\title{
The Balance and the Mind study: baseline data
}

Veronika van der Wardt, Pip Logan, Vicky Booth, Vicky Hood, Tahir Masud, Rowan Harwood

OBJECTIVES: The purpose of the study was to establish falls risk factors for people with mild cognitive impairment and mild dementia.

METHODS: Survey of 76 people recruited from Memory Clinics ( $n=56)$, Falls Services $(n=12)$, Community Geriatricians ( $n=4)$, Cognitive Stimulation Therapy groups $(n=2)$, and a Rehabilitation Unit $(n=2)$. The assessments included falls risk, the Falls Efficacy Scale International (FESi), the Montreal Cognitive Assessment (MoCA) as well as neuropsychological tests.

RESULTS: Mean age was 80.9 years (SD 6.5, age range: $67-94$ years). The sample included $45 \%$ men, $98 \%$ had a white ethnic background and 48\% lived alone. Mean MoCA score was 20.95 (SD 3.6), and 26 people (34\%) had between 1 and 24 falls (mean 3.04; SD 4.65). Regarding any fall participants had in the past, $41 \%$ sustained an injury, $36 \%$ needed medical attention and $32 \%$ had been admitted to hospital as a consequence of a fall.

The mean FESi score was 27 (SD 10.6) with 59\% scoring $>23$, which is associated with a high fear of falling. The mean Timed Up and Go score was 16.0 seconds with $44 \%$ scoring $\geq 13.5$ seconds, which is associated with high risk of falling. The mean Berg Balance score was 46 with $28 \%$ scoring $\leq 40$, which is associated with a $100 \%$ chance of future falls. All assessments were acceptable to the particpants.

CONCLUSIONS: The proportion of falls and falls risk factors was quite high in this population, which reflects findings from similar studies. Specialized falls prevention intervention should take these factors into account.

(max 250 words) 